\title{
A COMMUNITY STUDY OF CARRIERS IN EPIDEMIC POLIOMYELITIS ${ }^{1}$
}

\author{
By HERBERT A. WENNER ${ }^{2}$ AND ALBERT E. CASEY \\ (From the Department of Pediatrics, Yale University School of Medicine, New Haven, and \\ the Department of Pathology and Bacteriology, Louisiana State \\ University School of Medicine, New Orleans)
}

(Received for publication June 15, 1942)

Since 1937, simple methods for the detection of poliomyelitis virus in human stools ( 1 to 4 ) have proved useful in case finding. The effectiveness of these methods has broadened our ideas about the way the virus is distributed in familial $(5,6)$, institutional $(7)$, and community $(8,9)$ outbreaks of poliomyelitis. The evidence adduced has indicated that the virus can be found in human stools under specified conditions, viz., in paralytic, abortive, and ambulatory convalescent cases of poliomyelitis, and in presumably healthy contacts, during epidemic periods.

The next step should be to determine the distribution of the virus in the whole population at risk during natural epidemics of the disease. This has not as yet been reported. Opportunity for such a "herd study" occurred in the State of Alabama during the fall of $1941 .^{3}$ The present report concerns the examination of 176 of 181 persons, living in a defined community, affected in the extension of a widespread epidemic of poliomyelitis, to determine the carrier rate in a post-epidemic period.

\section{MATERIAL AND METHODS}

Clinical material. In the summer of 1941 , a widespread epidemic of poliomyelitis occurred in the state of Alabama. In mid-August, one of us (A. E. C.), already at work in the field, called attention to the presence of a heavy concentration of cases in the small town of Cordova (pop. 1670), Walker County, Alabama.4 Shortly thereafter, a geographically defined community on the eastern cor-

1 Aided by a grant from the National Foundation for Infantile Paralysis, Inc.

2 Frank Lusk Babbott, Jr. Fellow in Pediatrics.

${ }^{3}$ For the privilege of making these studies, we are indebted to the late Dr. J. N. Baker, former State Health Officer, Montgomery, Alabama; and especially to Dr. A. M. Waldrup, Walker County Health Officer, Jasper, Alabama. The assistance of these health officers and their staffs is gratefully acknowledged.

4 In the summer and fall of 1941, 871 reported cases of poliomyelitis occurred in the State of Alabama. Of these 871,125 cases with a 2 per cent mortality occurred in Walker County (pop. 67,000) Alabama. ridor of Cordova, containing most of the town's cases, was selected for study. Facilities for a combined clinical and laboratory study were provided, and after September 6, 1941, clinical histories of all, and stool specimens from 176 persons, were obtained.

Character of the community. The community selected for study, though situated on the outskirts of Cordova can be described as semi-rural, but over-crowded houses and outbuildings containing various domestic animals were close together. The houses were largely poor wooden structures. Surface privies of poor construction were almost universally present.

The inhabitants were mainly coal miners, and cottonmill workers.

A diagrammatic schema of the community studied is shown in Figure 1.

Course of epidemics. Six cases of poliomyelitis from this community were reported to the Walker County Health Department during July and August 1941. Their distribution appears in Figure 2.

Collection of stools. Between August 15 and August 25, 1941, one of us (A. E. C.) collected stools in 50 per cent glycerol from 6 children in the community, all suspected of having had poliomyelitis. Between September 6 and September 15, stools were obtained from 74 persons. Stools from 163 persons were collected before September 20 , and of the remaining 18,13 provided stools before October 6. Stools from 5 adults were not obtained. One other stool was collected on November 11.

Stools were obtained under fairly uniform conditions. In a house to house canvass, the essential articles, and careful instructions for collecting each stool specimen, were left with some responsible member. Infants' and young children's stools were collected in individual sterilized pots, and then transferred to a clean cardboard container. All other stools were deposited on wax paper, and then placed in the container. Stools were collected within 12 to 24 hour.s, stored in a refrigerator at $6^{\circ} \mathrm{C}$., and held there usually 1 to 5 days before etherization. A small number of stools remained in the ice-box for 10 to 20 days, and the glycerinated stools remained there for 50 to 80 days before extraction.

Preparation of inoculum. Roughly a 15 per cent aqueous stool suspension was divided into two fractions, (a) an untreated suspension for intra-nasal instillation, and (b) an etherized fraction (10 per cent ether by volume added to the 15 per cent stool suspension, which was allowed to stand in contact at ice-box temperature overnight) from which the middle layer was obtained after centrifugation for intraperitoneal inoculation. 


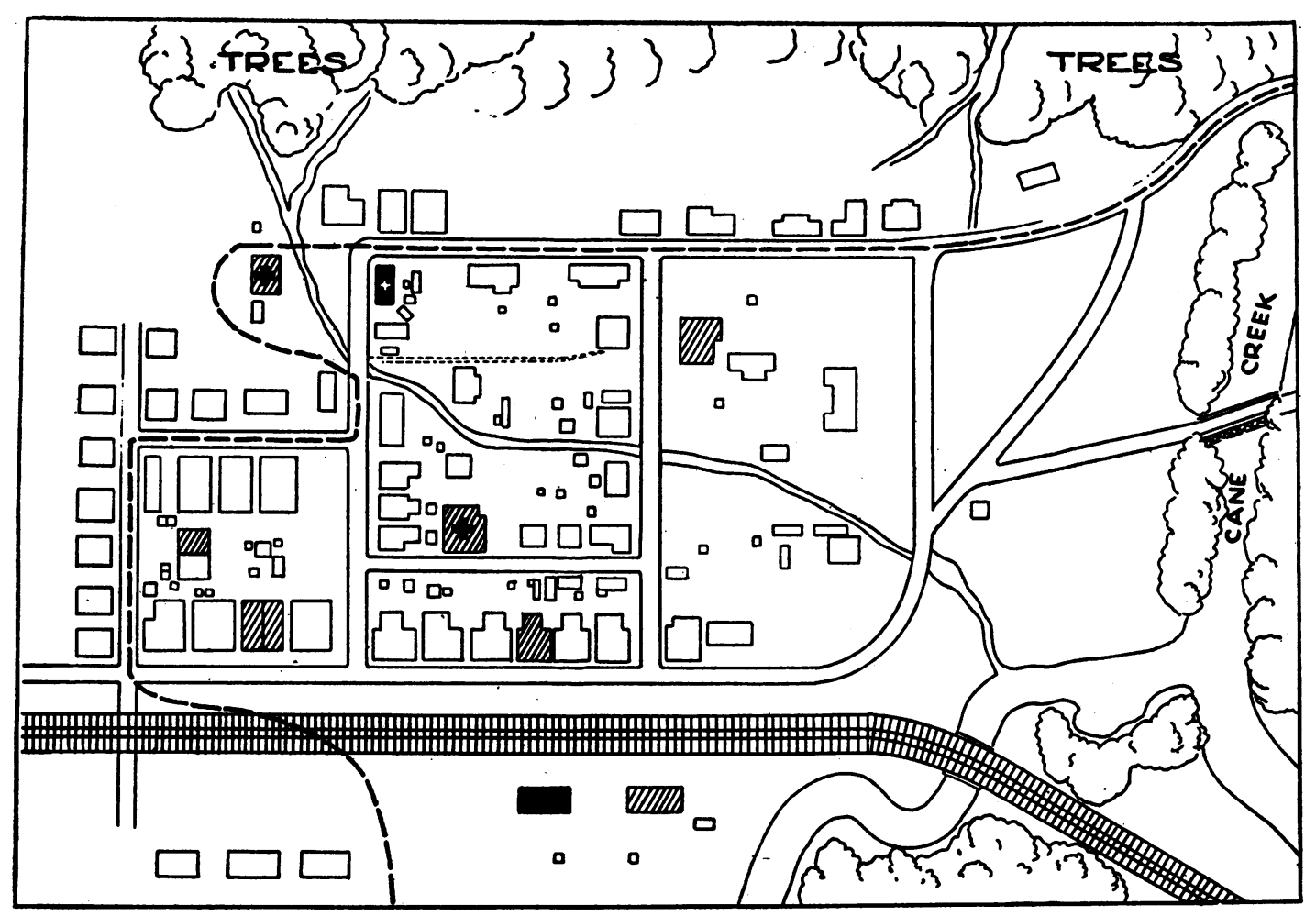

Fig. 1. Diagram of Community Studied

The community is outlined by the broken line and the lower and right hand margins of the figure; the area is roughly $1000 \times 600$ feet. The black blocks represent houses in which paralytic poliomyelitis occurred; the shaded blocks represent houses in which abortive poliomyelitis occurred. The residence of individuals providing positive stools is indicated by a cross.

Two types of inocula were used, (1) pooled stool inocula, i.e., $1.5 \mathrm{cc}$. of fraction (a), and $5.0 \mathrm{cc}$. of the etherized fraction $(b)$, each from 4 individuals in the same age group. These were pooled for use in one monkey (47 pools in all). (2) Individual stool inocula, vis., 6.0 cc. of fraction (a), and $20.0 \mathrm{cc}$. of fraction (b) were composed of stools from one person. These were

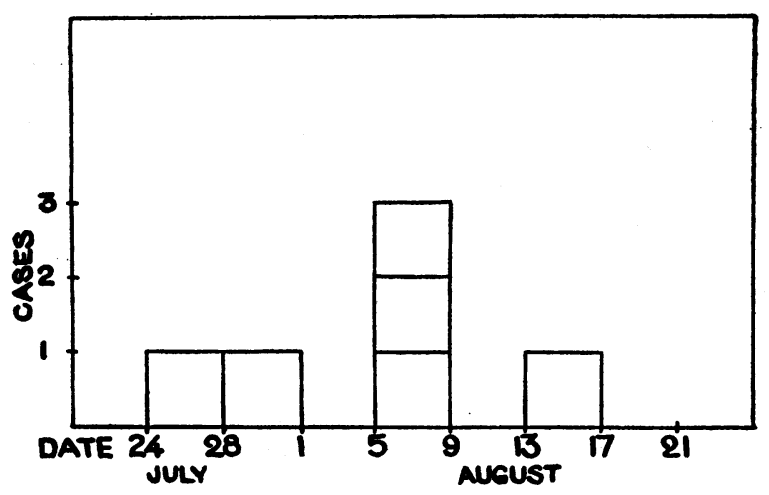

Fig. 2. Reported Cases of Poliomyelitis Which Occurred in the Community inoculated into at least one monkey (25 stools in all). Glycerinated stools were extracted in the same manner as raw stools.

Inoculation. The combined intraperitoneal and intranasal portals were used for inoculation. Each monkey received 10.0 to $20.0 \mathrm{cc}$. of the etherized fraction (b) once intraperitoneally; and also $2.0 \mathrm{cc}$. of raw stool extract, fraction (a), were dropped into the nasopharynx of each animal on each of 3 consecutive days.

Animals. Macaca mulatta weighing roughly 1.5 to 3.5 $\mathrm{kgm}$. were used. Inoculated animals were exercised daily, and daily rectal temperatures charted for 5 weeks unless the animal was killed earlier. Ten monkeys were reinoculated after 5 weeks of uneventful clinical observation.s All animals which showed symptoms of poliomyelitis, or remotely suggestive of poliomyelitis, were sacrificed at what appeared to be an appropriate time. All other monkeys were sacrificed at the end of the 5 week experimental period. Olfactory bulbs, midbrain, medulla, and cervical, thoracic, and lumbar regions of the spinal

- Monkeys used for second tests were tuberculin tested using $10.0 \mathrm{mgm}$. of human old tuberculin in the test dose. Only tuberculin negative animals were used. 
cord were preserved in 10 per cent formalin, and in $\mathbf{5 0}$ per cent glycerol.

Criteria for identification of poliomyelitis virus. The criteria adopted for the identification of the virus were: (1) the production of experimental poliomyelitis in monkeys, viz., after a variable incubation period, usually 5 to 15 days, the development of fever, excitement, tremor, weakness, and paralysis, the latter being generally associated with a fall in temperature; and (2) histological lesions typical of poliomyelitis in the lumbar and cervical cords.

Passage of the virus to a second monkey was accomplished with one strain, but was not considered to be an essential criterion under the conditions of the present study.

\section{RESULTS}

Accidental losses. Two monkeys died prematurely, one of purulent peritonitis, the other of an abscess of the abdominal wall. These tests were
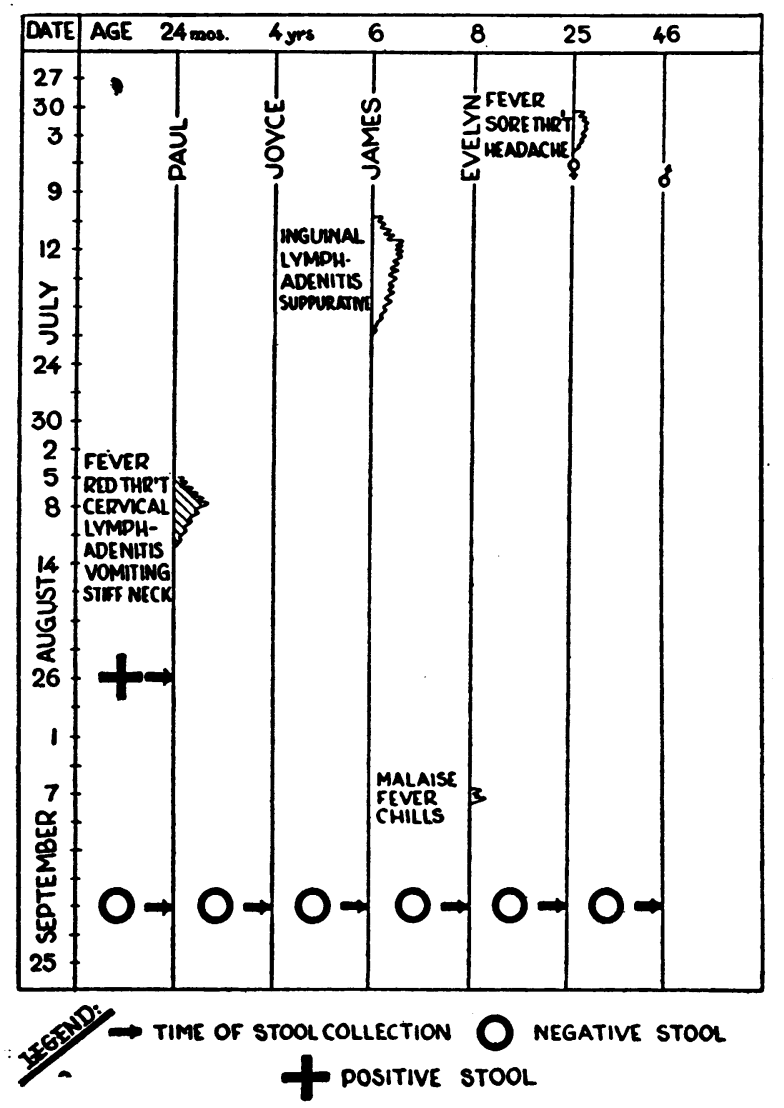

Fig. 3. Schematic Diagram of Family $H$

The vertical lines represent different individuals of the family; their ages appear at the top. The shaded area indicates the time of onset and course of an abortive attack of poliomyelitis; the open area indicates febrile illness. completed in other monkeys with negative results. One monkey died at the end of the experimental period, in the 5th week, of pulmonary tuberculosis.

Completed tests. One hundred and ninety stools from 176 individuals were subjected to 81 tests, of which 8 were positive. Three of these were from 47 pools; 4 of the 8 were from the subsequent fractionation of the pools, and the remaining positive was also from a single stool specimen. Altogether there were 3 individuals with positive tests. The results are amplified below.

The first child represents an abortive attack of poliomyelitis.

Family H (Figure 3). Paul H., male, aet. 2 years, one of 4 siblings. Birth history was uneventful. Whooping cough at 6 months was followed by pneumonia. He had recurrent pneumonia at 1 year, with prolonged convalescence during which time he had "colitis" with bloody diarrhea. On June 30, through July 4, 1941, his mother, aet. 25 years, had fever, headache, and sore throat. From July 10 to July 20, a brother, aet. 6 years, had suppurative inguinal lymphadenitis. On August 4, Paul H. was irritable, and refused to eat. The next day he was feverish, cried, and gagged frequently. The examining physician found fever, stiff neck, red throat, and cervical adenitis. On August 4, his neck was still stiff, and he vomited once. He was confined to bed for 6 days, but thereafter seemed well. A stool specimen from Paul $\mathrm{H}$. obtained August 26, and inoculated 77 days later into monkey A-69, was positive. A-69 developed fever on the 14th day, paralysis on the 18th day, and was sacrificed on the 20th day after inoculation. Typical histological lesions were found in the cord. Stools collected on September 18, and on October 6, provided negative results.

The second child represents a paralytic case.

FAMILY N (Figure 4). Manley N., male, aet. 3 years, one of 8 siblings. Birth history was uneventful He had whooping cough at 1 year. During 1940 and 1941, he had frequent upper respiratory infections with visible swelling of lymph nodes in the neck. A sister, aet. 14 years, from July 8 to July 11, 1941, had fever, vomiting, diarrhea, and headache. On August 13, Manley N. had rhinorrhea, and seemed feverish. After a day in bed, he felt well enough to get up and play with his siblings. He was fretful for the next few days, and on August 19, appeared listless and vomited. Two days later a physician found fever of $101^{\circ} \mathrm{F}$, a stiff neck, left cervical adenitis, a rapid pulse, and drowsiness. Paralysis of the left leg was observed on August 23. He was examined by one of us (H. A. W.) on December 4. No paralysis was detectable. Stools collected August 26, and September 8 , were positive. The first stool preserved in glycerine, and inoculated 77 days after collection, produced 


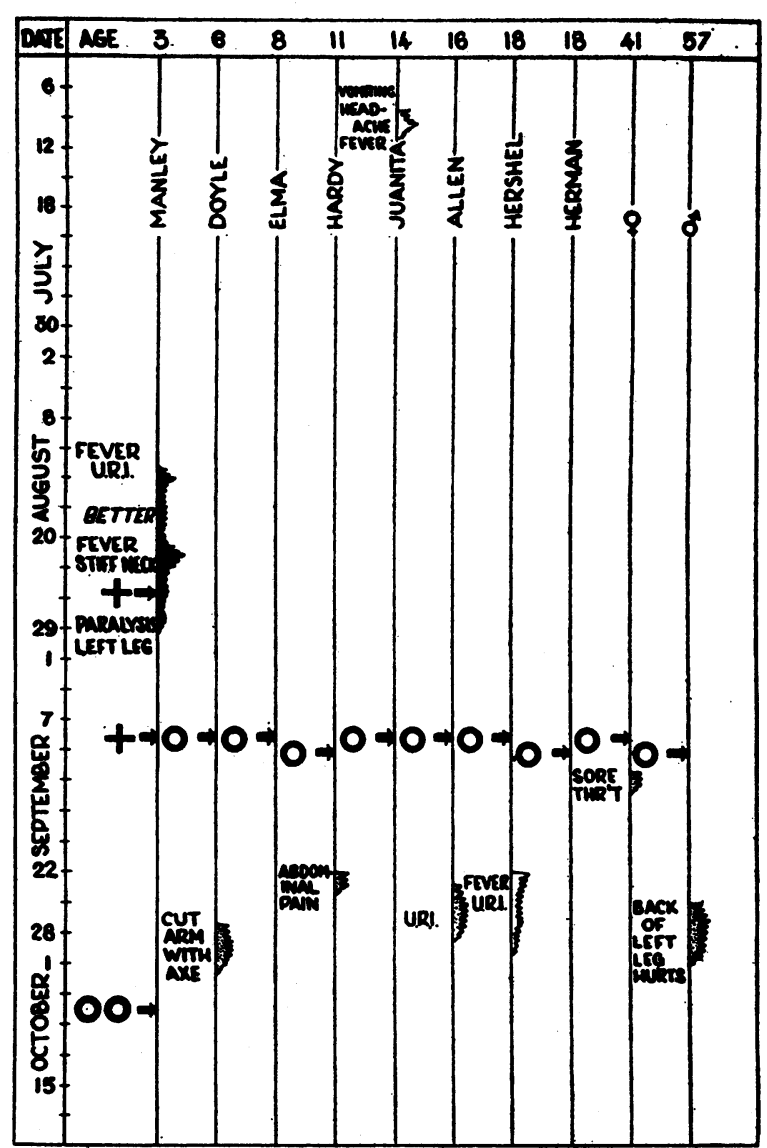

Fig. 4. Schematic Diagram of Family $\mathrm{N}$

The legends are the same as in Figure 3. The black area indicates the time of onset and course of a paralytic attack of poliomyelitis; the stippled areas indicate poorly defined illnesses.

fever in monkey A-70 on the 11th, and paralysis on the 14th day. The second stool produced fever in monkey A -45 on the 13th, and paralysis on the 15th day. The monkeys were sacrificed on the 18th and 19th days, and lesions typical of poliomyelitis, found in the cords of both. A stool collected October 6, 1941, was negative.

The third child with positive stools represents an ambulatory convalescent carrier, with a poorly defined antecedent illness.

FAMILY V (Figure 5). Lamarr V., male, aet. 6 years, one of 3 siblings. Birth history was uneventful. He had bronchopneumonia at 2 years, and whooping cough at 3 . A sibling, aet. 9 years, had fever, headache, and stomachache for 3 days beginning July 16, 1941. A physician believed him to have the prodromal signs of poliomyelitis. Another sibling, aet. 3 years, had 2 days of diarrhea in the first week of August. Lamarr V. was apparently well, except that his mother thought him fretful during the last week or 10 days in August. Stools collected September 8, and October 6 were positive. The first stool produced fever in monkey $A-47$ on the 10th day, and paralysis on the 11th day. The second stool produced fever in A-22 on the 14th day, and afterwards, marked ataxia and the development of weakness of the left leg on the 18th day were observed. The monkeys were sacrificed on the 14th and 19th days, and histological lesions typical of poliomyelitis found in both animals. A third stool obtained November 14, 1941, was negative.

To summarize, then, of 190 stools tested, 5 from 3 individuals ( 2 to 6 years of age) contained enough virus to produce experimental poliomyelitis in 8 rhesus monkeys. Subsequent stools from these individuals were tested until negative. The period of carriage of the virus in the stools was at least 42 days (Lamarr V.) and 26 days (Manley N.). None of the adults' stools were positive.

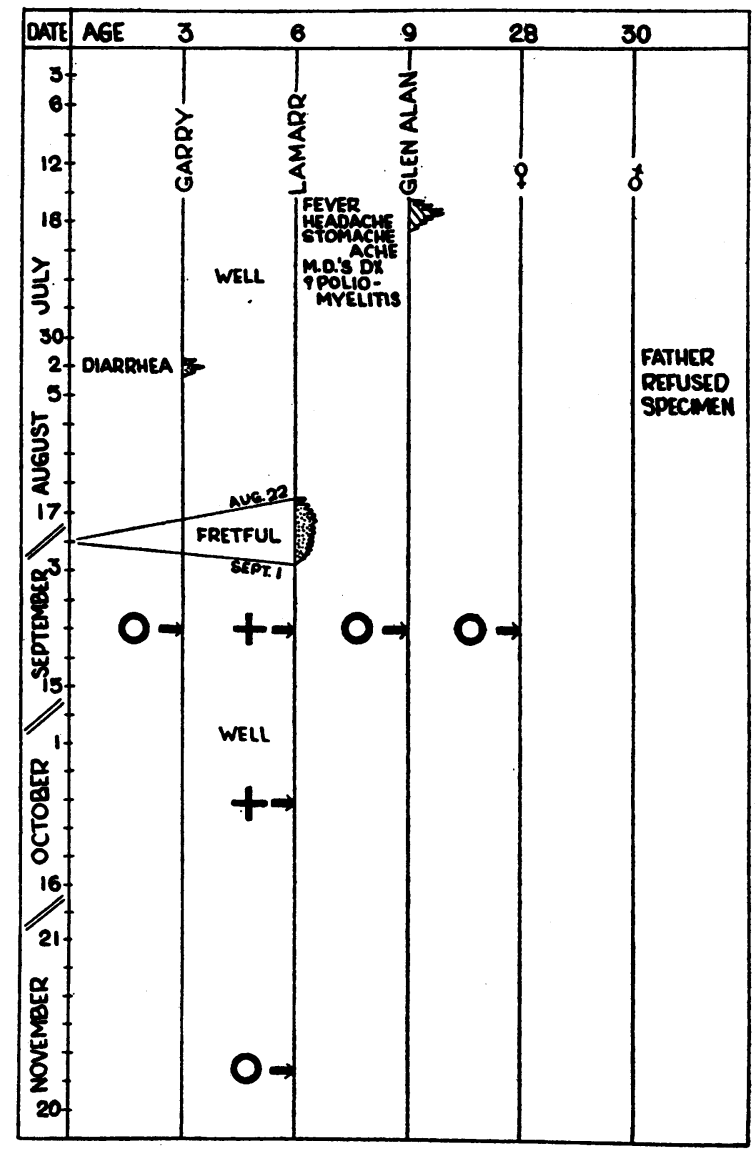

Fig. 5. Schematic Diagram of Family $V$

The legends are the same as in Figures 3 and 4. 


\section{CLINICAL RESULTS}

Classification of cases. Illnesses which occurred in the community during the summer were classified on the basis of the following terminology: (1) paralytic viz., cases with paralysis developing in association with the usual signs of poliomyelitis; (2) abortive, viz., cases affording a definite history of meningitic or myelitic signs (frequently affirmed by the attending physician), as well as fever, headache, vomiting, and other associated signs and symptoms; (3) suspected abortive, viz., cases with histories of febrile illnesses, usually associated with sore throat, vomiting, diarrhea, and headache, appearing in epidemic times, and not attributable to any other disease; (4) poorly defined illnesses, characterized in the main by the same symptoms as in the suspected abortive, with the exception that there was no known fever, and the illness was of brief duration, usually but for a day ; and (5) other diseases, viz., the exanthemata, tuberculosis, thrombophlebitis, and degenerative diseases.

In respect to time. The seasonal distribution of all of these illnesses (in both the adult and juvenile population) is shown in Figure 6. Poliomyelitis present in surrounding neighborhoods was first suspected in the community when one of the juvenile members became ill July 16, 1941. Thereafter, a wave of illness spread rapidly through the population, affecting the juvenile members worst. It reached the peak of its group incidence between August 1 and August 10, 1941.

In respect to age. Figure 7 delineates the clinical findings in the community from June 15 to September 15, 1941, compared to the population at risk, at various ages.

Adult population. A history of illness corresponding to one of the first four types in our classification was obtained from 33 of 117 adults. Two persons afforded histories only remotely suggestive of abortive poliomyelitis; 8 had febrile

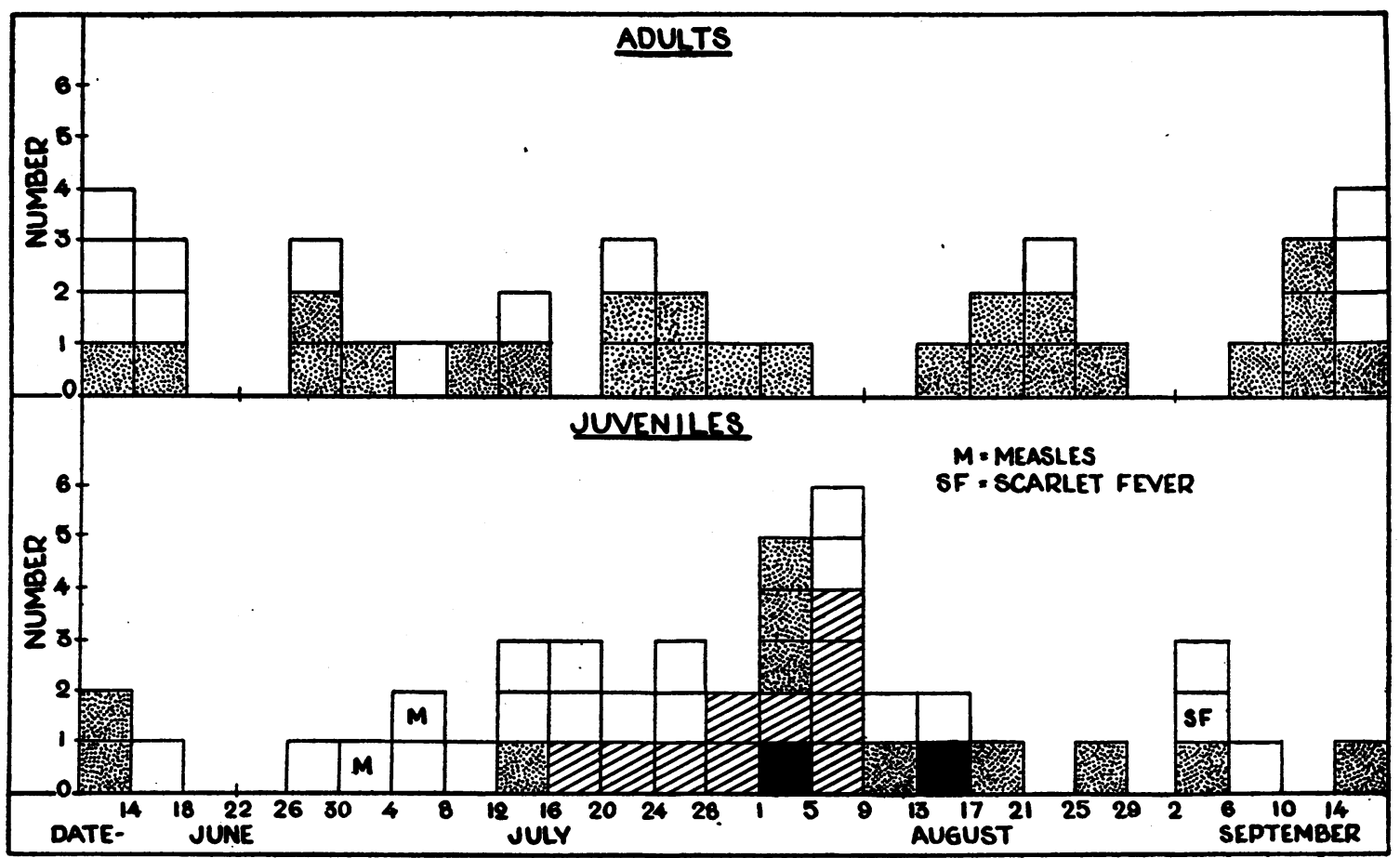

Fig. 6. The Seasonal Distribution of All Types of Illness in the Adult and Juvenile Populations DURING THE SUMmer of 1941

Black squares represent paralytic cases of poliomyelitis; shaded squares, abortive cases of poliomyelitis; open squares, febrile illnesses; and stippled squares, poorly defined ailments. 


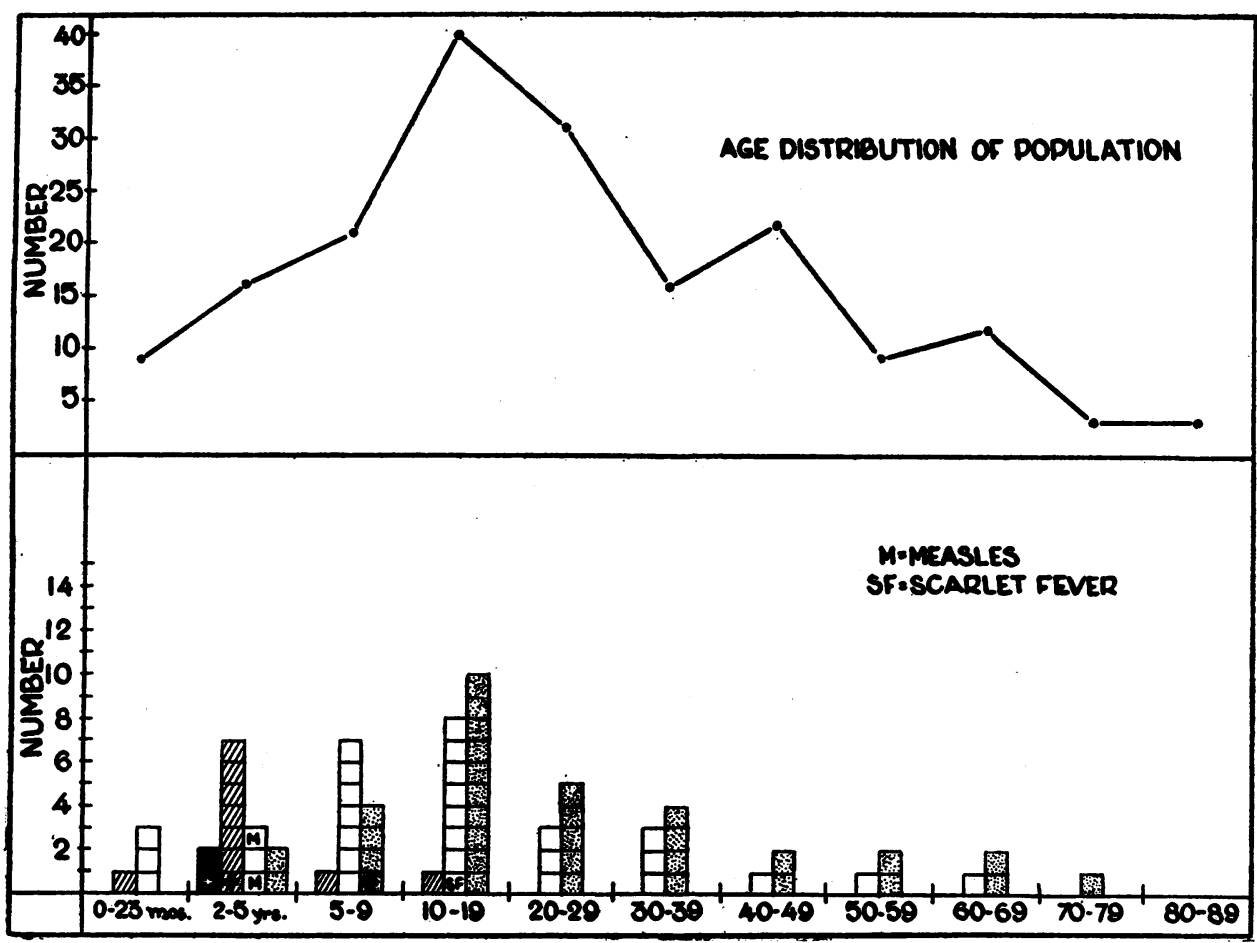

Fig. 7. Age Distribution of the Population at Risk (top panei) Compared with the Age Distribution of the Community Illness (lowkr panke) During 'the Pertod of STUDY

The small crosses indicate cases in which positive stool specimens were obtained. Otherwise the legends defining the blocked areas are the same as in Figure 6.

illnesses, usually with sore throat; and 23 had poorly defined ailments, characterized by rhinorrhea, abdominal pain, or headache. ${ }^{\circ}$

Juvenile population. In the juvenile group, 41 of 64 members had histories of one or more illnesses during the time from June 15 to September 15, 1941. Two children ( 2 and 3 years of age) had paralytic poliomyelitis; 10 individuals ( 1 to 12 years of age; average age, 4 years) had abortive poliomyelitis; and 17 persons ( 1 to 14 years of age; average age, 8 years) had febrile illnesses. Two children had measles, and one other scarlet fever. The remaining 9 of the 41 had ill-defined compaints characterized by headache, vomiting, diarrhea, or sore throat.

- Actually there were more adult illnesses than are indicated. One person had pulmonary tuberculosis; another acute thrombophlebitis; one other carcinoma, of which she died in November. Three persons received surgical care of major nature during the summer.

\section{ANALYSIS OF RESULTS}

In respect to method. When so few of our monkeys showed signs and symptoms of experimental poliomyelitis, it was suspected that pooling of stools was disadvantageous. To determine if this were so, stools from 10 of the 12 individuals in the community who provided histories of paralytic or abortive poliomyelitis were each given to at least 2 monkeys. The stools from these 10 persons had been negative previously in their respective pools. In the testing of these stools, 23 animals were used. All of these tests proved negative.

In respect to clinical material. Reference to Figure 7 shows that all of the paralytic, and 6 of the 10 abortive cases, occurred in the 2 to 5 year age group. Two of the 3 children with positive stools were in this age group; the third positive was 6 years of age. The greatest incidence of febrile illnesses occurred in the 10 to 19 , and 5 to 9 
year age groups, in that order. The large number of poorly defined illnesses occurring in the 10 to 19 year old age group is striking, although its meaning is not entirely clear.

The observed ratio of abortive to paralytic cases was 5 to 1 . The true ratio, considering some but not all of the 17 febrile illnesses as abortive cases, was probably greater than the observed ratio would indicate.

Critical examination of the illnesses in the adult group (Figure 6) shows in general that they do not have any real relationship, either as to type or to the seasonal distribution, when compared with the illnesses in the juvenile population. None of the adults had a positive stool test, notwithstanding the testing of 112 stools, almost twice the number tested in juvenile group.

In respect to time of illness and stool collection. The temporal relationship between the onset of illnesses, stool collections, and the results of stool tests in all members of the juvenile group is out- lined graphically in Figure 8. Attention has been called to the narrow time zone from July 16 to August 13, 1941, during which the onset of all recognized poliomyelitis cases occurred. At the time of stool collection, the 2 individuals with paralytic poliomyelitis were convalescent in the 4th and 5th week from onset, and the 10 abortive cases were in the 5 th to 7 th week.

Excepting the glycerinated stool specimens, ${ }^{7}$ 82 days, at most, elapsed between the onset of the first illness, July 16, and the collection of the last stool specimen on October 6. At least 24 days intervened from the onset of the last poliomyelitis case, August 13, and the beginning of stool collection, September 6. If one would consider only

IIt is of interest that 2 of the 6 glycerinated stool specimens which were kept in the refrigerator for 50 to 80 days were positive. Another glycerinated stool from an unidentified member of the community, which was kept in the refrigerator for 90 days produced experimental poliomyelitis in one of the monkeys.

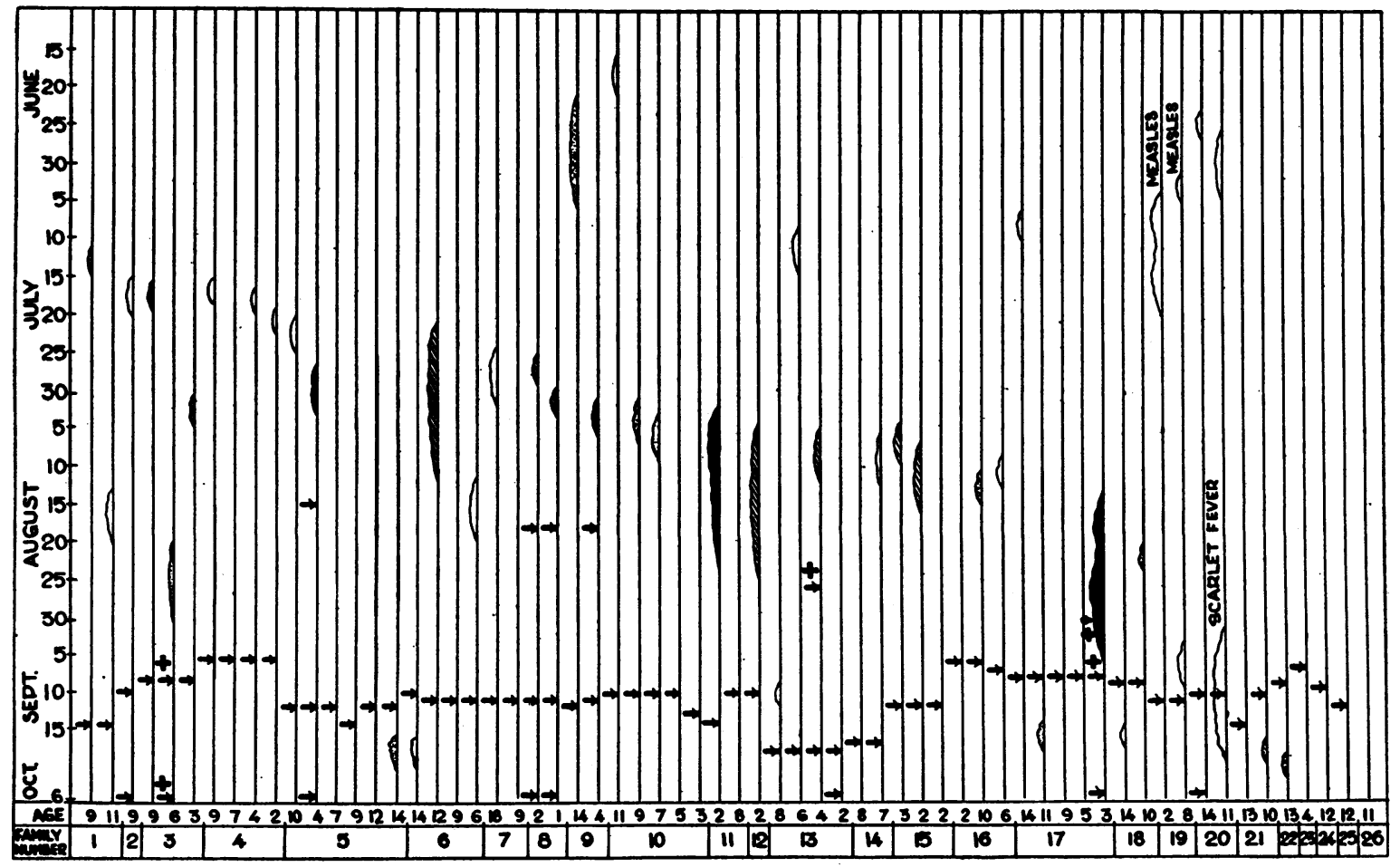

Fig. 8. Diagram of the Juvenile Members of the Community

All of the community children under 15 years of age appear as vertical lines. The onset and duration of illness in children ill during the summer is indicated by elevated areas appearing on the respective vertical lines. The type of illness in each individual is indicated by the same markings used in previous diagrams. The black arrows indicate the time of stool collection; the plus signs indicate positive tests. 
the children who had clinical poliomyelitis, we find that an average of 42 days elapsed from the time of onset of illness in each, and collection of their respective stools. At the most, it was $\mathbf{5 2}$ days and at the least, 26 days that passed.

In respect to time of illness and positive stools. Of the children with positive stools, 2 (Manley $\mathrm{N}$. and Lamarr V.) were among the last of those ill in the community during the epidemic period, viz., August 13 and August 25, respectively. The virus was detected in Manley $N$. on the 13th and 26th days after the onset of his illness, but not on the 54th day. Lamarr V.'s stools were positive on the 14th (if one arbitrarily dates the onset of his poorly defined illness on August 25th) and on the 32nd, but not on the 78th day. The third person yielding the virus (Paul H.), ill at the height of the epidemic, viz., August 5, was positive on the $21 \mathrm{st}$, but not on the 44th day from the beginning of his illness. Had we not had his stool in glycerol, collected August 26, and had we depended only on the September 18 stool, we would have missed detecting the virus in this individual.

Correlation of clinical and laboratory data. A rough correlation, considering the limitations of the present methods, can be drawn between the presence or absence of virus in stools, and the span of time elapsing between the onset of illness and stool collection in the members of the juvenile population. It would appear that there is a progressive decline in the number of carriers in the population corresponding with the decline of the disease.

Probably we began our tests too late to detect the maximum number of carriers. Our tests were begun 52 days after the onset of the epidemic, and 24 days after it had waned. The stool collections were between 82 and 24 days after the onset of the first and last recognized cases. In the majority of reports from the literature, the usual carrier period has been 3 weeks.

\section{DISCUSSION}

Past clinical epidemiological studies $(1,10,11)$ have emphasized a widespread distribution of poliomyelitis virus in the population at risk. It has been suggested that abortive cases of poliomyelitis outnumber at any time the paralytic cases, and that they, as well as healthy intermediary con- tacts, abet the spread of poliomyelitis by contact with susceptible individuals. Evidence obtained by use of the stool method $(5,6)$ has supported the clinical supposition that mild illnesses, occurring during epidemics, are abortive attacks of poliomyelitis. However the demonstration of a widespread distribution of the virus in "healthy contacts" by the use of these methods is quite recent.

In the past year, Piszczek, Shaughnessy, Zichis, and Levinson (8) and McClure and Langmuir (9) have described an unusually large number of healthy carriers. Some of these carriers were adults. This is not surprising in respect to a prevailing clinical suspicion, which it supports, that the virus is widespread among a large number of human hosts in epidemics. However, others (5, 6 ), in previous studies, have seldom been able to detect the virus in persons without a history of at least a poorly defined illness, and almost invariably children were the carriers of poliomyelitis virus.

In the present report, our results support the observations that the prolonged presence of the virus is usually associated with a current or antecedent illness, especially in children in the 2 to 5 year age group. Our results are partially at variance in two respects from the studies of Piszczek et al., and McClure and Langmuir: (1) None of our adults provided a positive stool test, and (2) all of our carriers had histories of past illnesses, mild in one to be sure, so that to attempt to differentiate between true "healthy carriers," and convalescent carriers is difficult at times.

There were, however, significant differences in method, bearing on the results of these other recent studies, which should be clarified. These are: (a) that the time of stool collection in respect to the onset of the epidemic was early as compared to the late time of stool collection in ours. In McClure and Langmuir's report, stools were collected from 5 to 24 days (average time 13 days) after the onset of the epidemic, compared with 49 and 82 days (average time 66 days) in ours. In the study of Piszczek et al., "stools were collected within the month after the onset of the disease" (8) in practically all the individuals examined. (b) Our study concerns the distribution of carriers in the whole population at risk. The other recent studies are concerned chiefly with the distri- 
bution of the virus in specified hosts. (c) The technical methods used differed in several details.

Further studies directed toward the detection of the virus of poliomyelitis are necessary to determine the natural distribution of poliomyelitis virus, and if, in the future, one were to detect the largest number of carriers possible, several things should be accomplished. These are: $(a)$ the collection of stools before the epidemic has waned, $(b)$ the use of a more susceptible test animal, i.e., $M$. $c y$ nomolgus, and (c) the use, if possible (and if they are developed), of more delicate technical methods. Probably the methods used in our study are inadequate, in so far as delicacy is concerned, for the detection of all carriers, but under the conditions of the present study, in a post-epidemic period, it seems unlikely that many carriers were missed from the population at risk. We may logically conclude therefore that the virus does not remain "active" in the stools of most persons for more than a few weeks.

\section{SUM MARY}

1. A community has been studied to determine the distribution of poliomyelitis in the population at risk in a post-epidemic period. Stools from 176 of 181 persons were tested to determine the carrier rate for poliomyelitis virus in the adult and juvenile population.

2. Stools containing the virus of poliomyelitis were detected in 3 children, from 2 to 6 years of age. None of the adults' stools were positive.

3. Though evidence of poliomyelitis was widespread in the human herd, only individuals ill at the end of the epidemic provided positive stools, indicating that the ease of detection of the virus in the herd at risk diminishes with the decline of the epidemic.
For valued advice given during this study, appreciation is gratefully expressed to the late Doctor James D. Trask.

\section{BIBLIOGRAPHY}

1. Kling, C., Petterssen, A., and Wernstedt, W., Experimental and pathological investigations. Communications Inst. méd. Etat, Stockholm, 1912, 3, 5.

2. Harmon, $P . H$., The use of chemicals as nasal sprays in prophylaxis of poliomyelitis in man. J. A. M. A., 1937, 109, 406, 1061.

3. Trask, J. D., Vignec, A. J., and Paul, J. R., Isolation of poliomyelitis virus from human stools. Proc. Soc. Exper. Biol. and Med., 1938, 38, 147.

4. Howe, H. A., and Bodian, D., Untreated human stools as a source of poliomyelitis virus. J. Infect. Dis., 1940, 66, 198.

5. Trask, J. D., Vignec, A. J., and Paul, J. R., Poliomyelitis virus in human stools. J. A. M. A., 1938, 111, 6.

6. Paul, J. R., and Trask, J. D., The virus of poliomyelitis in stools and sewage. J. A. M. A., 1941, 116, 493.

7. Kramer, S. D., Gilliam, A. G., and Molner, J. G., Recovery of the virus of poliomyelitis from the stools of healthy contacts in an institutional outbreak. Pub. Health Rep., 1939, 54, 1914.

8. Piszczek, E. A., Shaughnessy, H. J., Zichis, J., and Levinson, S. O., Acute anterior poliomyelitis, study of an outbreak in west suburban Cook County, Illinois: Preliminary report. J. A. M. A., 1941, 117, 1962. Also, personal communication from the senior author.

9. McClure, G. Y., and Langmuir, A. D., Search for carriers in an outbreak of acute anterior poliomyelitis in a rural community. The incidence of virus in feces. Am. J. Hyg., 1942, 35, 285.

10. Wickman, I., Acute Poliomyelitis, Nervous and Mental Disease Monograph, Series 16, New York, 1913. (Also previous papers.)

11. Paul, J. R., Salinger, R., and Trask, J. D., Studies on the epidemiology of poliomyelitis. I. Methods and criteria for the detection of abortive poliomyelitis. Am. J. Hyg., 1933, 17, 587. 